\title{
Solutions of fractional differential equations with $p$-Laplacian operator in Banach spaces
}

Jingjing $\operatorname{Tan}^{*}$ and Meixia Li

"Correspondence:

tanjingjing1108@163.com

School of Mathematics and

Information Science, Weifang

University, Weifang, P.R. China

\section{Springer}

\begin{abstract}
In this paper, we study the solutions for nonlinear fractional differential equations with $p$-Laplacian operator nonlocal boundary value problem in a Banach space. By means of the technique of the properties of the Kuratowski noncompactness measure and the Sadovskii fixed point theorem, we establish some new existence criteria for the boundary value problem. As application, an interesting example is provided to illustrate the main results.
\end{abstract}

Keywords: fractional differential equation; boundary value problem; $p$-Laplacian operator; noncompactness measure

\section{Introduction}

A $p$-Laplacian differential equation was first introduced by Leibenson [1] when he studied the turbulent flow in a porous medium. Converting this fundamental mechanics problem into the existence of solutions to the following $p$-Laplacian differential equation:

$$
\left(\varphi_{p}\left(u^{\prime}(t)\right)\right)^{\prime}=f(t, u(t)), \quad t \in(0,1)
$$

where $\varphi_{p}(s)=|s|^{p-2} s(p>1)$ is the $p$-Laplacian operator, its inverse function is denoted by $\varphi_{q}(s)$ with $\varphi_{q}(s)=|s|^{q-2} s$, and $p, q$ satisfy $\frac{1}{p}+\frac{1}{q}=1$, he solved the practical and significant theoretical problem. Then many important results relative to differential equation (1) with different initial conditions and boundary conditions have been obtained (e.g. [214]). Scholars now find that fractional-order models are more adequate than integer-order models for problems in various fields of science such as physics, fluid flows, electrical networks, and many other (e.g. [15-25]). Consequently, the research of fractional differential equations with $p$-Laplacian operator BVP has already become a focus in recent years, and it has developed very rapidly (e.g. [26, 27]).

The authors in [28] studied the existence of positive solutions for the nonlinear fractional equation with $p$-Laplacian operator

$$
\mathscr{D}_{0_{+}}^{\alpha}\left(\phi_{p}\left(\mathscr{D}_{0_{+}}^{\alpha} u(t)\right)\right)=f(t, u(t))
$$

(c) The Author(s) 2018. This article is distributed under the terms of the Creative Commons Attribution 4.0 International License (http://creativecommons.org/licenses/by/4.0/), which permits unrestricted use, distribution, and reproduction in any medium, provided you give appropriate credit to the original author(s) and the source, provide a link to the Creative Commons license, and indicate if changes were made. 
with the boundary conditions

$$
u(0)=0, \quad u(1)=a u(\xi), \quad D_{0_{+}}^{\alpha} u(0)=0,
$$

where $0<\alpha \leq 2,0<\eta \leq 1,0<\xi<1,0 \leq a \leq 1$. By using the Guo-Krasnosel'skii fixed point theorem and the Leggett-Williams theorem, some sufficient conditions for the existence positive solutions have been obtained. The authors in [29] considered the above equation with the boundary conditions

$$
u(0)=0, \quad \mathscr{D}_{0_{+}}^{\alpha} u(0)=0, \quad u(1)=a D_{0_{+}}^{\alpha} u(1) .
$$

Some existence and multiplicity results of positive solutions have been obtained. In [30], the authors also considered the same equation with the boundary conditions

$$
u(0)=0, \quad u(1)=a u(\xi), \quad D_{0_{+}}^{\alpha} u(0)=0, \quad \mathscr{D}_{0_{+}}^{\alpha} u(1)=b D_{0_{+}}^{\alpha} u(\eta),
$$

where $0 \leq a, b \leq 1$. They obtained the existence of at least one positive solution by means of the upper and lower solutions method.

As far as we know, few results have been obtained to the solutions of the fractional order differential equations with $p$-Laplacian operator nonlocal boundary value problem (BVP):

$$
\left\{\begin{array}{l}
-\mathscr{D}_{0+}^{\beta}\left(\varphi_{p}\left(D_{0+}^{\alpha} x\right)\right)(t)=f(t, x(t)), \quad 0<t<1, \\
x(0)=\theta, \quad \mathscr{D}_{0+}^{\alpha} x(0)=\theta, \\
\mathscr{D}_{0+}^{\gamma} x(1)=\sum_{i=1}^{m-2} \alpha_{i} D_{0+}^{\gamma} x\left(\xi_{i}\right),
\end{array}\right.
$$

in a Banach space $E$, where $\mathscr{D}_{0+}^{\alpha}, \mathscr{D}_{0+}^{\beta}$ and $\mathscr{D}_{0+}^{\gamma}$ are the standard Riemann-Liouville fractional derivatives, $\theta$ is the zero element of $E, 1<\alpha \leq 2,0<\beta, \gamma \leq 1, \alpha-\gamma-1 \geq 0$, $I=[0,1], f: I \times E \rightarrow E$ is continuous, $\alpha_{i} \geq 0(i=1,2, \ldots, m-2), 0<\xi_{1}<\xi_{2}<\cdots<\xi_{m-2}<1$, $\sum_{i=1}^{m-2} \alpha_{i} \xi_{i}^{\alpha-\gamma-1}<1$. We establish some existence of solutions to BVP (2). The technique relies on the properties of the Kuratowski noncompactness measure and the Sadovskii fixed point theorem. Obviously, BVP (2) is more general than the problems discussed in some recent literature (such as [28-30]). Firstly, the boundary conditions are nonlocal, which can cover the well-known Sturm-Liouville boundary conditions as a special case, so we generalize the results of [28]. Secondly, as we generalized the space from the scalar space to the abstract space, our work includes the results of [28-30].

The rest of this paper is organized as follows. In Section 2, we introduce some definitions and lemmas to prove our main results. In Section 3, the existence results of solutions to the BVP are discussed by using the properties of the Kuratowski measure of noncompactness and the Sadovskii fixed point theorem. Finally, one example is provided to illustrate our main results in Section 4.

\section{Preliminaries}

For convenience, we present here the necessary definitions and preliminary facts which are used throughout this paper. 
Definition 2.1 (see [31]) Let $\alpha>0$, the fractional integral of order $\alpha>0$ of a function $x:(0, \infty) \rightarrow R$ is given by

$$
l_{0+}^{\alpha} x(t)=\frac{1}{\Gamma(\alpha)} \int_{0}^{x} \frac{x(t)}{(t-s)^{1-\alpha}} d t
$$

provided that the right-hand side is pointwise defined on $(0, \infty)$.

Definition 2.2 (see [31]) The Riemann-Liouville standard fractional derivative of order $\alpha>0$ of a continuous function $x:[0, \infty) \rightarrow R$ is given by

$$
\mathscr{D}_{0+}^{\alpha} x(t)=\frac{1}{\Gamma(n-\alpha)}\left(\frac{d}{d t}\right)^{n} \int_{0}^{t} \frac{x(s)}{(t-s)^{\alpha-n+1}} d s
$$

where $n=[\alpha]+1,[\alpha]$ denotes the integer part of the real number $\alpha$, provided that the right-hand side integral is pointwise defined on $[0, \infty)$.

Proposition 2.1 (see $[32,33])$ Let $x$ be integrable,

(1) if $\alpha>0$, then

$$
\ell_{0+}^{\alpha} \mathcal{D}_{0+}^{\alpha} x(t)=x(t)+c_{1} t^{\alpha-1}+c_{2} t^{\alpha-2}+\cdots+c_{n} t^{\alpha-n},
$$

where $c_{i} \in R, i=1,2,3, \ldots, N, N$ is the smallest integer greater than or equal to $\alpha$.

(2) if $\beta>\alpha>0$, then

$$
\begin{aligned}
& D_{0+}^{\alpha} \ell_{0+}^{\beta} x(t)=\ell_{0+}^{\beta-\alpha} x(t), \\
& D_{0+}^{\alpha} \ell_{0+}^{\alpha} x(t)=x(t) .
\end{aligned}
$$

Definition 2.3 (Kuratowski measure of noncompactness, see [34]) Let $E$ be a real Banach space, $S$ be a bounded set in $E$, the Kuratowski measure of noncompactness of $S$ is given by

$$
\alpha(S)=\inf \left\{\delta>0: S=\bigcup_{i=1}^{m} S_{i}, \operatorname{diam}\left(S_{i}\right)<\delta, i=1,2, \ldots, m\right\},
$$

where $\operatorname{diam}\left(S_{i}\right)$ denotes the diameters of $S_{i}$.

Remark 2.1 From the definition, it is obvious that $0 \leq \alpha(S)<\infty$.

Definition 2.4 (k-set contraction operator, see [34]) Let $E_{1}$ and $E_{2}$ be real Banach spaces, $D \subset E_{1}, A: D \rightarrow E_{2}$ is a continuous and bounded operator. If there exists a constant $k \geq 0$ such that $\alpha(A(S)) \leq k \alpha(S)$ for any bounded set $S$ in $D$, then $A$ is called a k-set contraction operator.

Remark 2.2 When $k<1, A$ is called a strict set contraction operator. It is easy to prove that a strict set contraction operator is a condensing operator. 
Now, we denote

$$
Q(I)=\left\{x \in C[I, E]: \sup _{t \in I} \frac{\|x(t)\|}{1+t}<+\infty\right\}
$$

where $C[I, E]$ is the Banach space of a continuous function $x: I \rightarrow E$ with the norm $\|x\|_{C}=$ $\max _{t \in I}\|x(t)\|$. It is easy to see that $Q(I)$ is a Banach space with the norm $\|x\|_{Q}=\sup _{t \in I} \frac{\|x(t)\|}{1+t}$. The basic space used in this paper is $Q(I)$. The Kuratowski measure of noncompactness in $E, C[I, E]$ and $Q(I)$ are denoted by $\alpha_{E}(\cdot), \alpha_{C}(\cdot)$ and $\alpha_{Q}(\cdot)$, respectively.

The following properties of the Kuratowski noncompactness measure and the Sadovskii fixed point theorem are needed for our discussion.

Lemma 2.1 (see [35]) If $H \subset C[I, E]$ is bounded and equicontinuous, then $\alpha_{E}(H(t))$ is continuous on $I$ and $\alpha_{C}(H)=\max _{t \in I} \alpha_{E}(H(t)), \alpha_{E}\left(\int_{I} x(t) d t: x \in H\right) \leq \int_{I} \alpha_{E}(H(t)) d t$, where $H(t)=\{x(t): x \in H\}$ for each $t \in I$.

Lemma 2.2 (Sadovskii, see [34]) Let D be a bounded, closed and convex subset of the Banach space E. If the operator $A: D \rightarrow D$ is condensing, then $A$ has a fixed point in $D$.

\section{Main results}

For simplicity of presentation, we give some notations and list some conditions as follows:

$$
\begin{aligned}
& M=\sigma\left[1+\frac{m-1}{1-\sum_{i=1}^{m-2} \alpha_{i} \xi_{i}^{\alpha-\gamma-1}}\right], \quad \bar{M}=\max _{t \in I}\{a(t), b(t)\}, \\
& \sigma=\frac{(\Gamma(\beta))^{1-q}}{\Gamma(\alpha)}, \quad K_{r}=\{x \in E:\|x\| \leq r\}, \\
& K_{R}=\left\{x \in Q(I):\|x\|_{Q} \leq R\right\}, \quad K_{\rho}=\left\{x \in Q(I):\|x\|_{Q} \leq \rho\right\} .
\end{aligned}
$$

$\left(\mathrm{H}_{1}\right)$ There exist nonnegative functions $a, b \in C[0,1]$ such that

$$
\int_{0}^{t}\|f(s, x)\| d s \leq \varphi_{p}[a(t)\|x\|+b(t)], \quad \forall t \in I, x \in E
$$

and

$$
\int_{0}^{1}(1+t) a(t) d t<M, \quad \int_{0}^{1} b(t) d t<+\infty
$$

$\left(\mathrm{H}_{2}\right)$ For any $r>0,[\alpha, \beta] \subset I, f(t, x)$ is uniformly continuous on $[\alpha, \beta] \times K_{r}$ and $f(t, x) \geq 0$.

$\left(\mathrm{H}_{3}\right)$ For all $t \in[0,1]$, bounded subsets $W \subset E$, there exists a positive constant $l<\frac{1}{4 M}$ such that

$$
\alpha_{E}(f(s, W)) \leq \varphi_{p}\left(l \alpha_{E}(W)\right)
$$

In order to discuss the BVP, the preliminary lemmas are given in this section. 
Lemma 3.1 Given $y \in C[0,1]$ and $y \geq 0$. Then the following $B V P$

$$
\left\{\begin{array}{l}
-\mathscr{D}_{0+}^{\beta}\left(\varphi_{p}\left(D_{0_{+}}^{\alpha} x\right)\right)(t)=y(t), \quad 0<t<1, \\
x(0)=0, \quad \mathscr{D}_{0+}^{\alpha} x(0)=0, \\
\mathscr{D}_{0+}^{\gamma} x(1)=\sum_{i=1}^{m-2} \alpha_{i} \mathscr{D}_{0+}^{\gamma} x\left(\xi_{i}\right),
\end{array}\right.
$$

has a unique solution satisfying

$$
\begin{aligned}
x(t)= & -\sigma \int_{0}^{t}(t-s)^{\alpha-1} \varphi_{q}\left(\int_{0}^{s}(s-\tau)^{\beta-1} y(\tau) d \tau\right) d s \\
& +\sigma \frac{t^{\alpha-\gamma-1}}{1-\sum_{i=1}^{m-2} \alpha_{i} \xi_{i}^{\alpha-\gamma-1}} \int_{0}^{1}(1-s)^{\alpha-\gamma-1} \varphi_{q}\left(\int_{0}^{s}(s-\tau)^{\beta-1} y(\tau) d \tau\right) d s \\
& -\sigma \frac{t^{\alpha-\gamma-1}}{1-\sum_{i=1}^{m-2} \alpha_{i} \xi_{i}^{\alpha-\gamma-1}} \\
& \times \sum_{i=1}^{m-2} \alpha_{i} \int_{0}^{\xi_{i}}\left(\xi_{i}-s\right)^{\alpha-\gamma-1} \varphi_{q}\left(\int_{0}^{s}(s-\tau)^{\beta-1} y(\tau) d \tau\right) d s .
\end{aligned}
$$

Proof Step 1. From [36, Lemma 2.3], we know the following BVP

$$
\left\{\begin{array}{l}
-\mathscr{D}_{0+}^{\alpha} x(t)=y(t), \quad 0<t<1, \\
x(0)=0, \quad \mathscr{D}_{0+}^{\gamma} x(1)=\sum_{i=1}^{m-2} \alpha_{i} D_{0+}^{\gamma} x\left(\xi_{i}\right)
\end{array}\right.
$$

has a unique solution satisfying

$$
\begin{aligned}
x(t)= & -\frac{1}{\Gamma(\alpha)} \int_{0}^{t}(t-s)^{\alpha-1} y(s) d s \\
& +\frac{1}{\Gamma(\alpha)} \frac{t^{\alpha-\gamma-1}}{1-\sum_{i=1}^{m-2} \alpha_{i} \xi_{i}^{\alpha-\gamma-1}} \int_{0}^{1}(1-s)^{\alpha-\gamma-1} y(s) d s \\
& -\frac{1}{\Gamma(\alpha)} \frac{t^{\alpha-\gamma-1}}{1-\sum_{i=1}^{m-2} \alpha_{i} \xi_{i}^{\alpha-\gamma-1}} \sum_{i=1}^{m-2} \alpha_{i} \int_{0}^{\xi_{i}}\left(\xi_{i}-s\right)^{\alpha-\gamma-1} y(s) d s .
\end{aligned}
$$

Step 2. Let $u=\mathscr{D}_{0+}^{\alpha} x(t)$ and $v=\varphi_{p}(u)$. It is easy to know that $u=\varphi_{q}(v)$. By Proposition 2.1, the solution of the following initial value problem

$$
\left\{\begin{array}{l}
-D_{0+}^{\beta} v(t)=y(t), \quad 0<t<1, \\
v(0)=0
\end{array}\right.
$$

can be written as $v(t)=-\ell_{0+}^{\beta} y(t), t \in[0,1]$. Combining with the expression of $u$, we know that the solution of (3) satisfies

$$
\left\{\begin{array}{l}
-\mathscr{D}_{0+}^{\alpha} x(t)=\varphi_{p}^{-1}\left(-l_{0+}^{\beta} y(t)\right), \quad 0<t<1, \\
x(0)=0, \quad \mathscr{D}_{0+}^{\gamma} x(1)=\sum_{i=1}^{m-2} \alpha_{i} \mathscr{D}_{0+}^{\gamma} x\left(\xi_{i}\right) .
\end{array}\right.
$$


As we have stated in Step 1, we can easily get the solution of BVP (6) as follows:

$$
\begin{aligned}
x(t)= & \frac{1}{\Gamma(\alpha)} \int_{0}^{t}(t-s)^{\alpha-1} \varphi_{q}\left(-l_{0+}^{\beta} y(t)\right) d s \\
& -\frac{1}{\Gamma(\alpha)} \frac{t^{\alpha-\gamma-1}}{1-\sum_{i=1}^{m-2} \alpha_{i} \xi_{i}^{\alpha-\gamma-1}} \int_{0}^{1}(1-s)^{\alpha-\gamma-1} \varphi_{q}\left(-l_{0+}^{\beta} y(t)\right) d s \\
& +\frac{1}{\Gamma(\alpha)} \frac{t^{\alpha-\gamma-1}}{1-\sum_{i=1}^{m-2} \alpha_{i} \xi_{i}^{\alpha-\gamma-1}} \sum_{i=1}^{m-2} \alpha_{i} \int_{0}^{\xi_{i}}\left(\xi_{i}-s\right)^{\alpha-\gamma-1} \varphi_{q}\left(-l_{0+}^{\beta} y(t)\right) d s .
\end{aligned}
$$

Since $y(t) \geq 0, t \in[0,1]$, we have $\varphi_{p}^{-1}\left(-l_{0+}^{\beta} y(t)\right)=-\left(l_{0+}^{\beta} y(t)\right)^{q-1}$, which implies that the solution of (3) is given by (4).

The following lemma is a straightforward conclusion of Lemma 3.1.

Lemma 3.2 Suppose that condition $\left(\mathrm{H}_{1}\right)$ is satisfied. Then BVP (2) has a unique solution satisfying

$$
\begin{aligned}
x(t)= & \sigma \int_{0}^{t}(t-s)^{\alpha-1} \varphi_{q}\left(\int_{0}^{s}(s-\tau)^{\beta-1} f(\tau, x(\tau)) d \tau\right) d s \\
& +\frac{\sigma t^{\alpha-\gamma-1}}{1-\sum_{i=1}^{m-2} \alpha_{i} \xi_{i}^{\alpha-\gamma-1}} \int_{0}^{1}(1-s)^{\alpha-\gamma-1} \varphi_{q}\left(\int_{0}^{s}(s-\tau)^{\beta-1} f(\tau, x(\tau)) d \tau\right) d s \\
& -\frac{\sigma t^{\alpha-\gamma-1}}{1-\sum_{i=1}^{m-2} \alpha_{i} \xi_{i}^{\alpha-\gamma-1}} \\
& \times \sum_{i=1}^{m-2} \alpha_{i} \int_{0}^{\xi_{i}}\left(\xi_{i}-s\right)^{\alpha-\gamma-1} \varphi_{q}\left(\int_{0}^{s}(s-\tau)^{\beta-1} f(\tau, x(\tau)) d \tau\right) d s .
\end{aligned}
$$

Proof The proof is similar to Lemma 3.1, so we omit.

For any $x \in Q(I)$, we define the operator $T$ by

$$
\begin{aligned}
(T x)(t)= & -\sigma \int_{0}^{t}(t-s)^{\alpha-1} \varphi_{q}\left(\int_{0}^{s}(s-\tau)^{\beta-1} f(\tau, x(\tau)) d \tau\right) d s \\
& +\frac{\sigma t^{\alpha-\gamma-1}}{1-\sum_{i=1}^{m-2} \alpha_{i} \xi_{i}^{\alpha-\gamma-1}} \int_{0}^{1}(1-s)^{\alpha-\gamma-1} \varphi_{q}\left(\int_{0}^{s}(s-\tau)^{\beta-1} f(\tau, x(\tau)) d \tau\right) d s \\
& -\frac{\sigma t^{\alpha-\gamma-1}}{1-\sum_{i=1}^{m-2} \alpha_{i} \xi_{i}^{\alpha-\gamma-1}} \\
& \times \sum_{i=1}^{m-2} \alpha_{i} \int_{0}^{\xi_{i}}\left(\xi_{i}-s\right)^{\alpha-\gamma-1} \varphi_{q}\left(\int_{0}^{s}(s-\tau)^{\beta-1} f(\tau, x(\tau)) d \tau\right) d s .
\end{aligned}
$$

Remark 3.1 Lemma 3.2 indicates that the existence of solution to BVP (2) is equivalent to the existence of the fixed point of the operator $T$.

Lemma 3.3 Suppose that conditions $\left(\mathrm{H}_{1}\right)$ and $\left(\mathrm{H}_{2}\right)$ are satisfied. Then the operator $T$ : $Q(I) \rightarrow Q(I)$ is continuous and bounded. 
Proof Step 1. For any $x \in Q(I)$, we prove that $(T x)(t) \in Q(I)$. By condition $\left(\mathrm{H}_{1}\right)$, together with the definition of operator $T$, we have

$$
\begin{aligned}
&\left\|\frac{(T x)}{1+t}(t)\right\| \\
& \leq\left\|\frac{\sigma}{1+t} \int_{0}^{t}(t-s)^{\alpha-1} \varphi_{q}\left(\int_{0}^{s}(s-\tau)^{\beta-1} f(\tau, x(\tau)) d \tau\right) d s\right\| \\
& \quad+\left\|\frac{\sigma}{1+t} \frac{t^{\alpha-\gamma-1}}{1-\sum_{i=1}^{m-2} \alpha_{i} \xi_{i}^{\alpha-\gamma-1}} \int_{0}^{1}(1-s)^{\alpha-\gamma-1} \varphi_{q}\left(\int_{0}^{s}(s-\tau)^{\beta-1} f(\tau, x(\tau)) d \tau\right) d s\right\| \\
& \quad+\| \frac{\sigma}{1+t} \frac{t^{\alpha-\gamma-1}}{1-\sum_{i=1}^{m-2} \alpha_{i} \xi_{i}^{\alpha-\gamma-1}} \\
& \quad \times \sum_{i=1}^{m-2} \alpha_{i} \int_{0}^{\xi_{i}}\left(\xi_{i}-s\right)^{\alpha-\gamma-1} \varphi_{q}\left(\int_{0}^{s}(s-\tau)^{\beta-1} f(\tau, x(\tau)) d \tau\right) d s \| \\
& \leq \sigma \int_{0}^{1}(1-s)^{\alpha-1} \varphi_{q}\left(\int_{0}^{s}\|f(\tau, x(\tau))\| d \tau\right) d s \\
& \quad+ \frac{\sigma}{1-\sum_{i=1}^{m-2} \alpha_{i} \xi_{i}^{\alpha-\gamma-1}} \int_{0}^{1} \varphi_{q}\left(\int_{0}^{s}\|f(\tau, x(\tau))\| d \tau\right) d s \\
&+\frac{\sigma(m-2)}{1-\sum_{i=1}^{m-2} \alpha_{i} \xi_{i}^{\alpha-\gamma-1}} \int_{0}^{1} \varphi_{q}\left(\int_{0}^{s}\|f(\tau, x(\tau))\| d \tau\right) d s \\
& \leq M \int_{0}^{1} \varphi_{q}\left(\int_{0}^{s}\|f(\tau, x(\tau))\| d \tau\right) d s \\
& \leq M \int_{0}^{1}[a(s)\|x\|+b(s)] d s \\
& \leq M\left[\int_{0}^{1}(1+s) a(s) d s\|x\|_{Q}+\int_{0}^{1} b(s) d s\right] \\
&<+\infty
\end{aligned}
$$

This means that $(T x)(t)$ is well defined and $(T x)(t) \in Q(I)$ for any $x \in Q(I)$.

Step 2. It is time to show that $T$ is a bounded operator. For any $x \in B_{\rho}$, from (8), we get

$$
\left\|\frac{(T x)(t)}{1+t}\right\| \leq M \bar{M}(2 \rho+1)
$$

So $T$ maps bounded sets into bounded sets in $Q(I)$, it follows that $T$ is a bounded operator.

Step 3. It remains to show that $T$ is continuous on $Q(I)$. Let $x_{n}, x \in Q(I)$ with $\lim _{n \rightarrow+\infty}\left\|x_{n}-x\right\|_{Q} \rightarrow 0$. It is trivial to see that $\left\{x_{n}\right\}$ is a bounded subset of $Q(I)$. As a result, there exists a constant $\eta>0$ such that $\left\|x_{n}\right\|_{Q} \leq \eta$ for all $n \geq 1$. Taking limit, we see $\|x\|_{Q} \leq \eta$. Taking $\left(\mathrm{H}_{2}\right)$ into consideration, we know that for any $\varepsilon>0$, there exists $N>0$ such that

$$
\left\|f\left(s, x_{n}(s)\right)-f(s, x(s))\right\| \leq M^{\frac{1}{1-q}} \varepsilon^{\frac{1}{q-1}}, \quad \forall n \geq N, s \in I .
$$


According to (8), a routine computation gives rise to the inequality as follows:

$$
\begin{aligned}
& \left\|\frac{\left(T x_{n}\right)(t)}{1+t}-\frac{(T x)(t)}{1+t}\right\| \\
& \quad \leq \frac{M}{1+t}\left\{\int_{0}^{1} \varphi_{q}\left(\int_{0}^{s}\left\|f\left(\tau, x_{n}(\tau)\right)-f(\tau, x(\tau))\right\| d \tau\right) d s\right\} \\
& \quad \leq \frac{\varepsilon}{1+t} \\
& \quad<\varepsilon .
\end{aligned}
$$

It follows that $\left\|\frac{\left(T x_{n}\right)(t)}{1+t}-\frac{(T x)(t)}{1+t}\right\|_{Q}<\varepsilon$. Thus $T: Q(I) \rightarrow Q(I)$ is continuous. This completes the proof of Lemma 3.3 .

Lemma 3.4 Let condition $\left(\mathrm{H}_{1}\right)$ be satisfied and $V$ be a bounded subset of $Q(I)$. Then $\frac{(T V)(t)}{1+t}$ is equicontinuous on $[0,1]$.

Proof In fact, in the light of the boundedness of $V$, namely, for any $x \in V$, there exists $\tilde{\eta}>0$ such that $\|x\|_{Q} \leq \tilde{\eta}$. Without loss of generality, suppose that $t_{1}, t_{2} \in I$ with $t_{1}<t_{2}$ by means of the monotonicity of $\frac{(t-s)^{\alpha-1}}{1+t}$ in $t$ for $s<t$ and the mean value theorem. Combining with the definition of operator $T$, we have

$$
\begin{aligned}
& \left\|\frac{(T x)\left(t_{2}\right)}{1+t_{2}}-\frac{(T x)\left(t_{1}\right)}{1+t_{1}}\right\| \\
& \leq \sigma \| \frac{1}{1+t_{2}} \int_{0}^{t_{2}}\left(t_{2}-s\right)^{\alpha-1} \varphi_{q}\left(\int_{0}^{s}(s-\tau)^{\beta-1} f(\tau, x(\tau)) d \tau\right) d s \\
& -\frac{1}{1+t_{1}} \int_{0}^{t_{1}}\left(t_{1}-s\right)^{\alpha-1} \varphi_{q}\left(\int_{0}^{s}(s-\tau)^{\beta-1} f(\tau, x(\tau)) d \tau\right) d s \| \\
& +\frac{\sigma}{1-\sum_{i=1}^{m-2} \alpha_{i} \xi_{i}^{\alpha-\gamma-1}} \| \frac{t_{2}^{\alpha-\gamma-1}}{1+t_{2}} \int_{0}^{1}(1-s)^{\alpha-\gamma-1} \varphi_{q}\left(\int_{0}^{s}(s-\tau)^{\beta-1} f(\tau, x(\tau)) d \tau\right) d s \\
& -\frac{t_{1}^{\alpha-\gamma-1}}{1+t_{1}} \int_{0}^{1}(1-s)^{\alpha-\gamma-1} \varphi_{q}\left(\int_{0}^{s}(s-\tau)^{\beta-1} f(\tau, x(\tau)) d \tau\right) d s \| \\
& +\frac{\sigma}{1-\sum_{i=1}^{m-2} \alpha_{i} \xi_{i}^{\alpha-\gamma-1}} \\
& \times \| \frac{t_{2}^{\alpha-\gamma-1}}{1+t_{2}} \sum_{i=1}^{m-2} \alpha_{i} \int_{0}^{\xi_{i}}\left(\xi_{i}-s\right)^{\alpha-\gamma-1} \varphi_{q}\left(\int_{0}^{s}(s-\tau)^{\beta-1} f(\tau, x(\tau)) d \tau\right) d s \\
& -\frac{t_{1}^{\alpha-\gamma-1}}{1+t_{1}} \sum_{i=1}^{m-2} \alpha_{i} \int_{0}^{\xi_{i}}\left(\xi_{i}-s\right)^{\alpha-\gamma-1} \varphi_{q}\left(\int_{0}^{s}(s-\tau)^{\beta-1} f(\tau, x(\tau)) d \tau\right) d s \| \\
& \leq \sigma \| \frac{1}{1+t_{2}} \int_{0}^{t_{1}}\left(t_{2}-s\right)^{\alpha-1} \varphi_{q}\left(\int_{0}^{s}(s-\tau)^{\beta-1} f(\tau, x(\tau)) d \tau\right) d s \\
& +\frac{1}{1+t_{2}} \int_{t_{1}}^{t_{2}}\left(t_{2}-s\right)^{\alpha-1} \varphi_{q}\left(\int_{0}^{s}(s-\tau)^{\beta-1} f(\tau, x(\tau)) d \tau\right) d s \\
& -\frac{1}{1+t_{1}} \int_{0}^{t_{1}}\left(t_{1}-s\right)^{\alpha-1} \varphi_{q}\left(\int_{0}^{s}(s-\tau)^{\beta-1} f(\tau, x(\tau)) d \tau\right) d s \|
\end{aligned}
$$




$$
\begin{aligned}
& +\frac{\sigma}{1-\sum_{i=1}^{m-2} \alpha_{i} \xi_{i}^{\alpha-\gamma-1}}\left|\frac{t_{2}^{\alpha-\gamma-1}}{1+t_{2}}-\frac{t_{1}^{\alpha-\gamma-1}}{1+t_{1}}\right| \\
& \times\left\{\left\|\int_{0}^{1}(1-s)^{\alpha-\gamma-1} \varphi_{q}\left(\int_{0}^{s}(s-\tau)^{\beta-1} f(\tau, x(\tau)) d \tau\right) d s\right\|\right. \\
& \left.+\left\|\sum_{i=1}^{m-2} \alpha_{i} \int_{0}^{\xi_{i}}\left(\xi_{i}-s\right)^{\alpha-\gamma-1} \varphi_{q}\left(\int_{0}^{s}(s-\tau)^{\beta-1} f(\tau, x(\tau)) d \tau\right) d s\right\|\right\} \\
& \leq \sigma\left|\frac{t_{2}^{\alpha}}{1+t_{2}}-\frac{t_{1}^{\alpha}}{1+t_{1}}-\frac{\left(t_{2}-t_{1}\right)^{\alpha}}{1+t_{2}}\right|\left\|\int_{0}^{t_{1}} \varphi_{q}\left(\int_{0}^{s}(s-\tau)^{\beta-1} f(\tau, x(\tau)) d \tau\right) d s\right\| \\
& +\sigma \frac{1}{1+t_{2}}\left\|\int_{t_{1}}^{t_{2}}\left(t_{2}-s\right)^{\alpha-1} \varphi_{q}\left(\int_{0}^{s}(s-\tau)^{\beta-1} f(\tau, x(\tau)) d \tau\right) d s\right\| \\
& +\frac{\sigma(m-2)}{1-\sum_{i=1}^{m-2} \alpha_{i} \xi_{i}^{\alpha-\gamma-1}}\left|\frac{t_{2}^{\alpha-\gamma-1}}{1+t_{2}}-\frac{t_{1}^{\alpha-\gamma-1}}{1+t_{1}}\right| \\
& \times\left\|\int_{0}^{1}(1-s)^{\alpha-\gamma-1} \varphi_{q}\left(\int_{0}^{s}(s-\tau)^{\beta-1} f(\tau, x(\tau)) d \tau\right) d s\right\| \\
& \leq \sigma\left|\frac{t_{2}^{\alpha}}{1+t_{2}}-\frac{t_{1}^{\alpha}}{1+t_{1}}\right| \int_{0}^{1} \varphi_{q}\left(\int_{0}^{s}\|f(\tau, x(\tau))\| d \tau\right) d s \\
& +\sigma \int_{t_{1}}^{t_{2}} \varphi_{q}\left(\int_{0}^{s}\|f(\tau, x(\tau))\| d \tau\right) d s \\
& +\frac{\sigma(m-2)}{1-\sum_{i=1}^{m-2} \alpha_{i} \xi_{i}^{\alpha-\gamma-1}}\left|\frac{t_{2}^{\alpha-\gamma-1}}{1+t_{2}}-\frac{t_{1}^{\alpha-\gamma-1}}{1+t_{1}}\right| \int_{0}^{1} \varphi_{q}\left(\int_{0}^{s}\|f(\tau, x(\tau))\| d \tau\right) d s \\
& \leq \sigma \bar{M}\left(2^{\alpha-1}+\frac{m-2}{1-\sum_{i=1}^{m-2} \alpha_{i} \xi_{i}^{\alpha-\gamma-1}}\right)(2 \tilde{\eta}+1)\left(t_{2}-t_{1}\right) \\
& +\sigma \bar{M}(2 \tilde{\eta}+1)\left(t_{2}-t_{1}\right) \\
& =\sigma \bar{M}\left(1+2^{\alpha-1}+\frac{m-2}{1-\sum_{i=1}^{m-2} \alpha_{i} \xi_{i}^{\alpha-\gamma-1}}\right)(2 \tilde{\eta}+1)\left(t_{2}-t_{1}\right) \text {. }
\end{aligned}
$$

Let

$$
\delta=\left[\sigma \bar{M}\left(1+2^{\alpha-1}+\frac{m-2}{1-\sum_{i=1}^{m-2} \alpha_{i} \xi_{i}^{\alpha-\gamma-1}}\right)(2 \tilde{\eta}+1)\right]^{-1} \cdot \frac{\varepsilon}{2} .
$$

It follows from (12) that

$$
\left\|\frac{(T x)\left(t_{2}\right)}{1+t_{2}}-\frac{(T x)\left(t_{1}\right)}{1+t_{1}}\right\|<\varepsilon
$$

For the case of $t_{1} \geq t_{2}$, after a tedious computation similar to the one used in the case of $t_{1} \leq t_{2}$, we can also get (13). This ensures that $\frac{(T V)(t)}{1+t}$ is equicontinuous on $[0,1]$. The proof of Lemma 3.4 is finished.

The existence of solution to BVP (2) is as follows.

Theorem 3.1 Let conditions $\left(\mathrm{H}_{1}\right)-\left(\mathrm{H}_{3}\right)$ be satisfied. Then the BVP has at least one solution belonging to $Q(I)$. 
Proof From Remark 3.1, the main point of our argument is to show that the operator $T$ has a fixed point in $Q(I)$.

Step 1. Take

$$
R>\int_{0}^{1} b(s) d s \cdot\left[M^{-1}-\int_{0}^{1}(1+s) a(s) d s\right]^{-1} .
$$

We first prove that $T K_{R} \subset K_{R}$. In fact, for any $x \in K_{R}$ and $t \in I$, by (8), we have

$$
\begin{aligned}
\left\|\frac{(T x)(t) \|}{1+t}\right\| & \leq M \int_{0}^{1} \varphi_{q}\left(\int_{0}^{s}\|f(\tau, x(\tau))\| d \tau\right) d s \\
& \leq M\left[\int_{0}^{1}(1+s) a(s) d s\|x\|_{D}+\int_{0}^{1} b(s) d s\right] \\
& \leq M\left\{\int_{0}^{1}(1+s) a(s) d s R+R\left[M^{-1}-\int_{0}^{1}[(1+s) a(s)] d s\right]\right\}^{-1} \\
& <R .
\end{aligned}
$$

Thus, from Lemma 3.3, $T K_{R} \subset K_{R}$ follows.

Step 2. We show that $T$ is a strict set contraction operator. Let $D=\overline{c o}_{Q}\left(T K_{R}\right)$, i.e., $D$ is the convex closure of $T K_{R}$ in $Q(I)$. Clearly, $D$ is a nonempty, bounded, convex and closed subset of $K_{R}$. By Lemma 3.4, we see $\frac{\left(T K_{R}\right)(t)}{1+t}$ is equicontinuous on $I$, it follows that $\frac{T(D)(t)}{1+t}$ is equicontinuous on $I$. By means of the definition of $D$, it is trivial to see that $D \subset K_{R}$ and $T K_{R} \subset D$. According to Lemma 3.3, we know that $T: D \rightarrow D$ is bounded and continuous. In addition, it is apparent from $\left(\mathrm{H}_{2}\right)$ that $\{f(s, x(s)): x \in D\}$ is equicontinuous on $I$. Taking $\left(\mathrm{H}_{3}\right)$ and Lemma 2.1 into consideration, for any $t \in I$ and $U \subset D$, we have

$$
\begin{aligned}
& \alpha_{E}\left(\frac{(T U)(t)}{1+t}\right) \\
& \leq \sigma\left[\int_{0}^{1}(1-s)^{\alpha-1} \varphi_{q}\left(\int_{0}^{s} \alpha_{E}(\{f(\tau, x(\tau): x \in U)\}) d \tau\right) d s\right. \\
&+\int_{0}^{1} \frac{(1-s)^{\alpha-\gamma-1}}{1-\sum_{i=1}^{m-2} \alpha_{i} \xi_{i}^{\alpha-\gamma-1}} \varphi_{q}\left(\int_{0}^{s} \alpha_{E}(\{f(\tau, x(\tau): x \in U)\}) d \tau\right) d s \\
&\left.+\frac{m-2}{1-\sum_{i=1}^{m-2} \alpha_{i} \xi_{i}^{\alpha-\gamma-1}} \int_{0}^{1} \varphi_{q}\left(\int_{0}^{s} \alpha_{E}(\{f(\tau, x(\tau): x \in U)\}) d \tau\right) d s\right] \\
& \leq M \int_{0}^{1} \varphi_{q}\left(\int_{0}^{s} \alpha_{E}(\{f(\tau, x(\tau): x \in U)\}) d \tau\right) d s \\
&= M \int_{0}^{1} \varphi_{q}\left(\int_{0}^{s} \alpha_{E}(\{f(\tau, V)\}) d \tau\right) d s \\
& \leq M l \alpha_{E}(V),
\end{aligned}
$$

where $V=\{x(\tau): \tau \in I, x \in U\}$. For any given $\varepsilon>0$, we partition $U$ as follows:

$$
U=\bigcup_{i=1}^{n} U_{i}, \quad \operatorname{diam}\left(U_{i}\right)<\alpha_{D}(V)+\frac{\varepsilon}{5}, \quad i=1,2, \ldots, n
$$


Moreover, for any $x_{i} \in U_{i}$, there exists a partition $0=t_{0}<t_{1}<\cdots<t_{m}=1$ such that

$$
\left\|x_{i}(s)-x_{i}(t)\right\|<\frac{\varepsilon}{5}, \quad t, s \in\left[t_{j-1}, t_{j}\right] .
$$

Let $V_{i j}=\left\{x(t): x \subset U_{i}, t \in\left[t_{j-1}, t_{j}\right]\right\}$. It is easy to see that $V_{i j}$ is a partition of $V$, that is, $V=\bigcup_{i=1}^{n} \bigcup_{j=1}^{m} V_{i j}$. Owing to the partition of $V$, for any $x, y \subset U_{i}, t, s \in\left[t_{j-1}, t_{j}\right]$, we get

$$
\begin{aligned}
\operatorname{diam} V_{i j} & \leq\|x(s)-y(t)\| \\
& \leq\left\|x(s)-x_{i}(s)\right\|+\left\|x_{i}(s)-x_{i}(t)\right\|+\left\|x_{i}(t)-y(t)\right\| \\
& \leq(1+s)\left\|x-x_{i}\right\|_{D}+\frac{\varepsilon}{5}+(1+t)\left\|x_{i}-y\right\|_{D} \\
& \leq 4 \operatorname{diam} U_{i}+\frac{\varepsilon}{5} \\
& <4 \alpha_{D}(U)+\varepsilon .
\end{aligned}
$$

So, $\alpha_{E}(V)<4 \alpha_{D}(U)+\varepsilon$, due to $\varepsilon$ being arbitrary, we obtain

$$
\alpha_{E}(V) \leq 4 \alpha_{D}(U)
$$

By substituting (17) into (15), we have

$$
\alpha_{E}\left(\frac{(T U)(t)}{1+t}\right) \leq 4 M l \alpha_{D}(U)
$$

Taking the least upper bound of $\alpha_{E}\left(\frac{(T U)(t)}{1+t}\right)$ when $t$ is in the set of $I$, applying [11, Lemma 2.6], we know that

$$
\alpha_{D}(T U)=\sup _{t \in I} \alpha_{E}\left(\frac{(T U)(t)}{1+t}\right), \quad \forall U \subset D
$$

where

$$
\frac{(T U)(t)}{1+t}=\left\{\frac{(T x)(t)}{1+t}: x \in U, t \in I \text { is fixed }\right\} \subset D .
$$

Take $L=4 M l$. From (15), (18) and (19), we get

$$
\alpha_{D}(T U) \leq L \alpha_{D}(U)
$$

Obviously, $0 \leq L<1$, that is, $T$ is a strict set contraction operator from $D$ to $D$. Obviously, $T$ is condensing too. It follows from Lemma 2.2 that $T$ has at least one fixed point in $D$, that is, BVP (2) has at least one solution in $Q(I)$.

Remark 3.2 If $E=[0, \infty)$, as a special case of Theorem 3.1, we can obtain the following result.

Corollary 3.1 Let $\left(\mathrm{H}_{1}\right)-\left(\mathrm{H}_{3}\right)$ be satisfied and $f \in C[[0,1] \times[0, \infty),[0, \infty)]$. Then BVP $(2)$ has at least one solution in $Q(I)$.

Proof Letting $E=[0, \infty)$ in Theorem 3.1, we get the desired result. 


\section{Illustrative example}

Let $E=l_{\infty}=\left\{x=\left(x_{1}, x_{2}, \ldots, x_{n}, \ldots\right), \sup _{n}\left|x_{n}\right|<+\infty, t \in I\right\}$. It is easy to see that $E$ is a Banach space with the norm $\|x\|=\sup _{n}\left|x_{n}\right|$. We consider the following nonlocal fractional differential equations BVP:

$$
\left\{\begin{array}{l}
-\mathscr{D}_{0+}^{\frac{1}{2}}\left(\varphi_{\frac{3}{2}}\left(\mathcal{D}_{0+}^{\frac{3}{2}} x_{n}\right)\right)(t)=\left[\frac{x_{n}(t)}{16(1+t)\left(1+t^{2}\right)}+\frac{\sin (t)+\sqrt{\left|\sin \left(x_{n+1}(t)\right)\right|^{2}}}{42 n^{2} e^{\sqrt{t}}}\right]^{\frac{1}{2}}, \\
x_{n}(0)=0, \quad \mathscr{D}_{0+}^{\frac{3}{2}} x_{n}(0)=0 \\
\mathscr{D}_{0+}^{\frac{1}{4}} x_{n}(1)=\frac{1}{4} \mathscr{D}_{0+}^{\frac{1}{4}} x_{n}\left(\frac{1}{4}\right)+\frac{1}{2} \mathscr{D}_{0+}^{\frac{1}{4}} x_{n}\left(\frac{3}{4}\right) .
\end{array}\right.
$$

BVP (20) can be regarded as a problem with the form of BVP (2), where

$$
\begin{aligned}
& f(t, x)=\left(f_{1}(t, x), f_{2}(t, x), \ldots, f_{n}(t, x), \ldots\right), \\
& f_{n}(t, x)=\left[\frac{x_{n}(t)}{16(1+t)\left(1+t^{2}\right)}+\frac{\sin (t)+\sqrt{\left|\sin \left(x_{n+1}(t)\right)\right|^{2}}}{42 n^{2} e^{\sqrt{t}}}\right]^{\frac{1}{2}} .
\end{aligned}
$$

Clearly, $\alpha=\frac{3}{2}, \beta=\frac{1}{2}, \gamma=\frac{1}{4}, \sum_{i=1}^{m-2} \alpha_{i}=0.642<1$ and

$$
\int_{0}^{t}\left\|f_{n}(s, x)\right\| d s \leq \varphi_{\frac{3}{2}}\left(\frac{1}{16(1+t)\left(1+t^{2}\right)}+\frac{1}{42 e^{\sqrt{t}}}\right) .
$$

Take $l=0.02, a(t)=\frac{1}{16(1+t)\left(1+t^{2}\right)}+\frac{1}{42 e^{\sqrt{t}}}, b(t)=\frac{1}{42 e^{\sqrt{t}}}$. In view of $\Gamma\left(\frac{1}{2}\right) \approx 1.772$ and $\Gamma\left(\frac{3}{2}\right) \approx$ 0.8862 , by a simple computation, we have

$$
\begin{aligned}
& M=\frac{\Gamma(\beta)^{1-q}}{\Gamma(\alpha)}\left(1+\frac{m-1}{1-\sum_{i=1}^{m-2} \alpha_{i}}\right)=9.589 \\
& \int_{0}^{1}[(1+t) a(t)] d t=0.0693<M^{-1} \\
& \int_{0}^{1} b(t) d t=0.0126<+\infty
\end{aligned}
$$

Therefore, all the conditions of Theorem 3.1 are satisfied. Consequently, we infer that (20) has at least one solution.

\section{Acknowledgements}

This project is supported by Shandong Provincial Natural Science Foundation (Grant No. ZR2017LA002), Weifang Science and Technology Development Projects (Grant No. 2017GX025) and Doctoral Research Foundation of Weifang University (Grant No. 2017BS02).

Competing interests

The authors declare that they have no competing interests.

Authors' contributions

All authors contributed equally to this work. All authors read and approved the final manuscript.

\section{Publisher's Note}

Springer Nature remains neutral with regard to jurisdictional claims in published maps and institutional affiliations. 


\section{References}

1. Leibenson, LS: General problem of the movement of a compressible fluid in a porous medium. Izv. Akad. Nauk Kirg. SSR, Ser. Biol. Nauk 9, 7-10 (1983)

2. Samko, SG, Kilbas, AA, Marichev, Ol: Fractional Integral and Derivatives (Theory and Applications). Gordon and Breach, Switzerland (1993)

3. Krasnosel'skii, MA: Positive Solutions of Operator Equations. Noordhoff Gronigen, Netherlands (1964)

4. Lakshmikantham, V, Vatsala, AS: General uniqueness and monotone iterative technique for fractional differential equations. Appl. Math. Lett. 21, 828-834 (2008)

5. Fan, $\mathrm{XD}$, Cheng, $\mathrm{CZ}$, Wang, $\mathrm{HJ}$ : Sensitivity analysis for vector equilibrium problems under functional perturbations. Numer. Funct. Anal. Optim. 35, 564-575 (2013)

6. Rudin, W: Functional Analysis, 2nd edn. International Series in Pure and Applied Mathematics. McGraw-Hill, New York (1991)

7. Wei, ZL: Positive solution of singular Dirichlet boundary value problems for second order differential equation system. J. Math. Anal. Appl. 328, 1255-1267 (2007)

8. Zhang, XG, Liu, LS, Wu, YH: Positive solutions of nonresonance semipositone singular Dirichlet boundary value problems. Nonlinear Anal. 68, 97-108 (2008)

9. Balasubramaniam, P, Vembarasan, V, Senthilkumar, T: Approximate controllability of impulsive fractional integro-differential systems with nonlocal conditions in Hilbert space. Numer. Funct. Anal. Optim. 35, 177-197 (2013)

10. Lakshmikantham, V, Vatsala, AS: Basic theory of fractional differential equations. Nonlinear Anal. 69, 2677-2682 (2008)

11. Chen, HB, Li, PL: Three-point boundary value problems for second-order ordinary differential equations in Banach spaces. Comput. Math. Appl. 56, 1852-1860 (2008)

12. Guo, DJ, Lakshmikantham, V, Liu, X: Nonlinear Integral Equations in Abstract Spaces. Kluwer Academic, Dordrecht (1996)

13. Liu, YS: Positive solutions of boundary value problems for nonlinear singular differential equations in Banach spaces. Acta Math. Sin. 47, 131-140 (2004)

14. Cui, YJ, Zou, YM: Positive solutions of nonlinear singular boundary value problems in abstract spaces. Nonlinear Anal. $69,287-294(2008)$

15. Xu, XJ, Jiang, DQ, Yuan, CJ: Multiple positive solutions to singular positone and semipositone Dirichlet-type boundary value problems of nonlinear fractional differential equations. Nonlinear Anal. 74, 5685-5696 (2011)

16. Agarwal, RP, Meehan, M, O'Regan, D: Fixed Point Theory and Applications. Cambridge Univ. Press, Cambridge (2001)

17. $\mathrm{Lu}, \mathrm{HL}, \mathrm{Han}, \mathrm{ZL}, \mathrm{Sun}, \mathrm{S}, \mathrm{Liu}, \mathrm{J}$ : Existence on positive solutions for boundary value problems of nonlinear fractional differential equations with P-Laplacian. Adv. Differ. Equ. 2013, 30 (2013)

18. Kilbas, AA, Srivastava, HM, Trujillo, JJ: Theory and Applications of Fractional Differential Equations. North-Holland Mathematics Studies, vol. 204. Elsevier Science, Amsterdam (2006)

19. Wu, WQ, Zhou, XB: Eigenvalue of fractional differential equations with $p$-Laplacian operator. Discrete Dyn. Nat. Soc. 2013, 1-8 (2013)

20. Zhang, K: Nontrivial solutions of fourth-order singular boundary value problems with sign-changing nonlinear terms. Topol. Methods Nonlinear Anal. 40, 53-70 (2012)

21. Zhang, K: Existence of sign-changing solutions for some asymptotically linear three-point boundary value problems. Nonlinear Anal. 70(7), 2796-2805 (2009)

22. Zhang, K: On sign-changing solution for some fractional differential equations. Bound. Value Probl. 2017, 59 (2017)

23. Webb, JRL, Zima, M: Multiple positive solutions of resonant and non-resonant nonlocal boundary value problems. Nonlinear Anal. 71, 1369-1378 (2009)

24. Dix, JG, Karakostas, GL: A fixed point theorem for s-type operators on Banach spaces and its applications to boundary-value problems. Nonlinear Anal. 71, 3872-3880 (2009)

25. Nyamoradi, N, Baleanu, D, Agarwal, RP: Existence and uniqueness of positive solutions to fractional boundary value problem with nonlinear boundary conditions. Adv. Differ. Equ. 2013, 266 (2013)

26. Liu, XP, Jia, M, Gei, WG: The method of lower and upper solutions for mixed fractional four-point boundary value problem with p-Laplacian operator. Appl. Math. Lett. 65, 56-62 (2017)

27. Jia, M, Liu, XP: Multiplicity of solutions for integral boundary value problems of fractional differential equations with upper and lower solutions. Appl. Math. Comput. 232, 313-323 (2014)

28. Wang, JH, Xiang, HJ, Liu, ZL: Positive solutions for three-point boundary value problems of nonlinear fractional differential equations with $p$-Laplacian. Far East J. Appl. Math. 37(1), 33-47 (2009)

29. Chain, G: Positive solutions for boundary value problem of fractional differential equation with $p$-Laplacian operator Bound. Value Probl. 2012, 18 (2012)

30. Wang, JH, Xiang, HJ: Upper and lower solutions method for a class of singular fractional boundary value problems with P-Laplacian operator. Abstr. Appl. Anal. 2010, Article ID 971824 (2010)

31. Yang, X, Wei, ZL, Dong, W: Existence of positive solutions for the boundary value problem of nonlinear fractional differential equations. Commun. Nonlinear Sci. Numer. Simul. 17(1), 85-92 (2012)

32. Miller, KS, Ross, B: An Introduction to the Fractional Calculus and Fractional Differential Equations. Wiley, New York (1993)

33. Podlubny, I: Fractional Differential Equations. Mathematics in Science and Engineering. Academic Press, New York (1999)

34. Guo, DJ: Nonlinear Functional Analysis. Shandong Science Technology Publishing House, Jinan (1985)

35. Aubin, JP, Ekeland, I: Applied Nonlinear Analysis. John Wiley \& Sons, New York (1984)

36. $L i, C F, L o u, X N$, Zhou, Y: Existence of positive solutions of the boundary value problem for nonlinear fractional differential equations. Comput. Math. Appl. 59(3), 1363-1375 (2010) 